\title{
On the energy release rate and the $J$-integral for 3-D crack configurations
}

\author{
H. G.de LOREN Z I \\ General Electric Company, Corporate Research and Development, Schenectady, New York 12301, USA
}

\begin{abstract}
In this paper an analytical expression for the energy release rate has been derived and put in a form suitable for a numerical analysis of an arbitrary 3-D crack configuration. The virtual crack extension method can most conveniently be used for such a derivation. This method was originally developed from finite element considerations and the resulting expressions were, therefore, based on the finite element matrix formulation [1-5]. In this paper the derivation of the energy release rate leads to an expression which is independent of any specific numerical procedure. The formulation is valid for general fracture behavior including nonplanar fracture and shear lips and applies to elastic materials as well as materials following the deformation theory of plasticity. The body force effect is also included. For 3-D fracture problems it is of advantage to use both an average and a local form of the energy release rate and definitions for both forms are suggested. For certain restrictions on the crack geometry it is shown that the energy release rate reduces to the 3-D form of the $J$-integral.
\end{abstract}

\section{Introduction}

The calculation of the energy release rate by the virtual crack extension method was introduced independently by Hellen $[1,3]$ and Parks $[2,4,5]$. The method was developed from a purely numerical standpoint and was based on the calculation of the released energy when a crack in a finite element model was extended a small amount $\Delta a$. Using finite element arguments Parks also showed that this formulation of the energy release rate was equal to the path independent $J$-integral when the 2-D constant strain triangles were used in the finite element model [2]. Both Parks and Hellen formulated the method for 3-D as well as 2-D problems [2,3] and while [1-3] were derived for linear elastic material models, Parks later also extended the method to include nonlinear material behavior and large displacement formulations $[4,5]$.

Because of the finite element formulation of the virtual crack extension method, the resulting expressions were not analytical expressions for the energy release rate, but were based on the finite element matrix formulation. In this paper the virtual crack extension method will be used to derive the energy release rate for a general 3-dimensional crack configuration not from a finite element, but from a continuum mechanics viewpoint. The material may be completely anisotropic and it is assumed to be hyperelastic so that the derivation will be valid for elastic materials as well as materials following the deformation theory of plasticity. The derivation is not restricted to any particular mode of fracture, but applies to a general fracture behavior including nonplanar fracture with shear lips. The loading will, however, be restricted to be monotonic and for simplicity the crack faces are assumed to be traction free.

The resulting analytical expression for the energy release rate is completely 
general and not tied to a particular application like the finite element method. It is, however, also extremely efficient in finite element applications and has been used to calculate the energy release rate for general 3-D configurations with nonlinear material behavior [6]. For planar fracture with the crack extending in the plane of the crack it is shown that the expression for the energy release rate will reduce to the 3dimensional form of the $J$-integral.

\section{Problem formulation}

The purpose of the present investigation is to calculate the energy released when a crack in a body is advanced a small increment $\Delta a$. Let, therefore, the deformation states before and after the crack increment be represented by two cracked bodies which are identical in outer shape, but have cracks of slightly different lengths. Let the crack front in each body be described by the 3-dimensional vectors $a_{\alpha}(s)$ and $\bar{a}_{i}(s)$ where the spatial variation of the crack front is described by the parameter $s$. We shall in the following refer to the two bodies as configuration I and configuration II, respectively, and shall measure all quantities in configuration $I$ in the Cartesian coordinate system $x_{\alpha}$ and all quantities in configuration II in the Cartesian coordinate system $y_{i}$ (Fig. 1). The Greek indices will in the following always be used for quantities defined in the $x$-system and Latin indices for quantities defined in the $y$-system. For brevity the summation convention for repeated indices will be used throughout, i.e.

$$
f_{\alpha} x_{\alpha}=f_{1} x_{1}+f_{2} x_{2}+f_{3} x_{3}
$$

The two configurations are also subjected to the surface tractions $F_{\alpha}$ and $\bar{F}_{i}$ and the body forces $f_{\alpha}$ and $\bar{f}_{i}$, respectively.

We shall now assume the existence of a coordinate mapping that maps configuration I into configuration II in such a way that the outer boundaries, the crack faces, and the crack front in configuration I are mapped into the outer boundary, the crack faces, and the crack front of configuration II. This mapping is assumed to be one-to-one, so that a specific point in each configuration corresponds to one and only
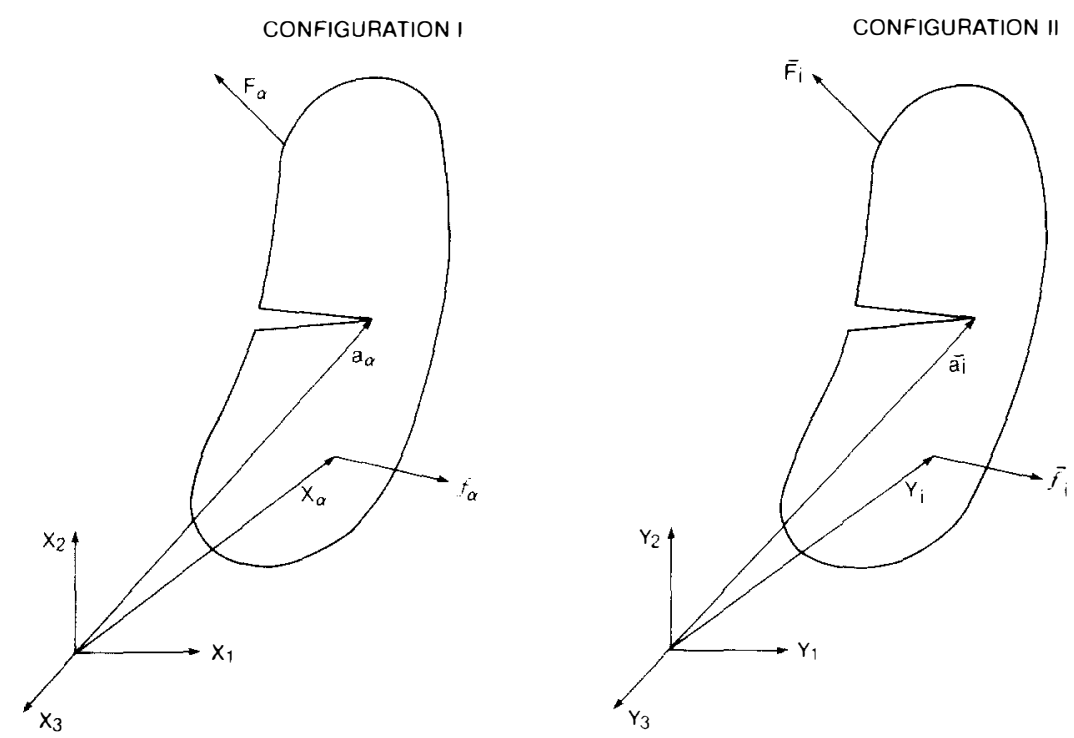

Figure 1. Crack configuration before and after crack extension. 
one point in the other configuration. It is also assumed that the mapping and the unique inverse mapping, which maps configuration II into configuration I, are sufficiently differentiable.

It should be pointed out that these mappings are not physical deformations of configuration I into configuration II and vice versa, but purely coordinate mappings. The material points located at the crack front in configuration I will, therefore, not be located at the crack front in configuration II. The reason for introducing the mappings is solely to facilitate the comparison of the singular stress and strain fields in the two bodies.

\section{Relationship between the two crack configurations}

Since the crack in configuration II is slightly longer than in configuration I, we can for each point on the crack front set

$$
\bar{a}_{i}=\delta_{i \alpha}\left(a_{\alpha}+\Delta a_{\alpha}\right)
$$

where the crack extension, $\Delta a_{\alpha}$, is "small" compared to other dimensions of the body and $\delta_{i \alpha}$ is a "shifter" which shifts a vector quantity in the $x$-system into the $y$-system. It should be noted that it will not be necessary to make any restrictions on $a_{\alpha}$ and $\Delta a_{\alpha}$ nor to make any assumptions about straight crack fronts or flat fracture. The derivations will, therefore, be equally valid for shear fracture and for cracks growing off at an angle to the original crack plane.

Since the difference in crack length between configuration I and II is small, we can make the restriction on the mapping from I to II that the point $x_{\alpha}$ is mapped into the point $\bar{y}_{i}$ according to

$$
\bar{y}_{i}\left(x_{j}\right)=\delta_{i \alpha}\left(x_{\alpha}+\Delta x_{\alpha}\left(x_{\beta}\right)\right)
$$

where $\Delta x_{\alpha}$ is a "small" quantity which is a function of the position $x_{\beta}$. By differentiating we get

$$
\frac{\partial \bar{y}_{i}}{\partial x_{\beta}}=\delta_{i \beta}+\delta_{i \alpha} \frac{\partial \Delta x_{\alpha}}{\partial x_{\beta}}
$$

and by neglecting all higher order terms we obtain the Jacobian determinant for the transformation in Eqn. (3)

$$
\operatorname{det}(J)=\operatorname{det}\left(\frac{\partial \bar{y}_{i}}{\partial x_{\beta}}\right) \approx 1+\frac{\partial \Delta x_{\alpha}}{\partial x_{\alpha}}
$$

By multiplying (4) on both sides with $\partial x_{\beta} / \partial \bar{y}_{j}\left(\delta_{i \epsilon}-\delta_{i \delta}\left(\partial \Delta x_{\epsilon} / \partial x_{\delta}\right)\right)$ and utilizing the fact that $\left(\partial \bar{y}_{i} / \partial x_{\beta}\right)\left(\partial x_{\beta} / \partial \bar{y}_{j}\right)=\delta_{i j}$ we get the inverse expression

$$
\frac{\partial x_{\epsilon}}{\partial \bar{y}_{j}} \approx \delta_{j \epsilon}-\delta_{j \delta} \frac{\partial \Delta x_{\epsilon}}{\partial x_{\delta}}
$$

We will now assume that the displacement in configuration II, $\bar{u}_{i}$, can be related to the displacements in configuration $\mathrm{I}, \boldsymbol{u}_{\alpha}$, by

$$
\bar{u}_{i}\left(\bar{y}_{i}\right)=\delta_{i \alpha}\left(u_{\alpha}\left(x_{\beta}\right)+\Delta u_{\alpha}\left(x_{\beta}\right)\right)
$$

where $\Delta u_{\alpha}$ is small compared to $u_{\alpha}$. Again it should be noted that (7) does not give a relationship between displacements of corresponding material points, but of points which have the same "relative" position to the crack tip. As stated in the previous section, we are dealing with coordinate mappings of points in the $x$-system into points in the $y$-system. A material point at $x_{\alpha}$ in configuration I will, therefore, not 
correspond to the material point at $\bar{y}_{i}$ given by (3), but rather to the point given by

$$
y_{i}=\delta_{i \alpha} x_{\alpha}
$$

A comparison of (3) and (8) reveals that $y_{i}=\bar{y}_{i}-\delta_{i \alpha} \Delta x_{\alpha}$, and the displacement of the material point $y_{i}$, which corresponds to the material point $x_{\alpha}$ in configuration $\mathrm{I}$, is, therefore, given by

$$
\bar{u}_{i}\left(y_{j}\right)=\bar{u}_{i}\left(\bar{y}_{j}-\delta_{j \alpha} \Delta x_{\alpha}\right) \approx \bar{u}_{i}\left(\bar{y}_{j}\right)-\left.\frac{\partial \bar{u}_{i}}{\partial \bar{y}_{j}}\right|_{\text {at } \bar{y}_{j}} \delta_{j \alpha} \Delta x_{\alpha}
$$

Using (7) and (6) we obtain by neglecting all higher order terms

$$
\bar{u}_{i}\left(\delta_{j \beta} x_{\beta}\right)=\delta_{i \alpha}\left(u_{\alpha}\left(x_{\beta}\right)+\Delta u_{\alpha}^{m}\left(x_{\beta}\right)\right)
$$

where the increment in displacement for the corresponding material point, $\Delta u_{\alpha}^{m}$, is given by

$$
\Delta u_{\alpha}^{m}=\Delta u_{\alpha}-\frac{\partial u_{\alpha}}{\partial x_{\beta}} \Delta x_{\beta}
$$

This expression is valid everywhere in the interior of configuration I and as the boundary is approached, except at the crack tip where $\partial u_{\alpha} / \partial x_{\beta}$ may become unbounded.

Since we require the outer boundaries (excluding the crack faces) to be identical in configurations I and II, we see that $\Delta x_{\beta}$ in (3) is equal to zero on these boundaries. The increment in physical displacement, $\Delta u_{\alpha}^{m}$, is, therefore, equal to $\Delta u_{\alpha}$ on the outer boundary.

\section{Energy considerations}

Let configuration I be subjected to the body forces $f_{i}$ and the surface tractions $F_{i}$ and configuration II to the corresponding loadings $\bar{f}_{i}$ and $\bar{F}_{i}$. The crack faces are, however, assumed to be traction free. The first law of thermodynamics states that the work performed on a body is equal to the increase in its internal energy, and if the two configurations are free from internal stresses in the undeformed states we get for each of them

$$
\begin{aligned}
& \int_{S}\left\{\int_{0}^{u_{\beta}} F_{\alpha} \mathrm{d} u_{\alpha}\right\} \mathrm{d} s+\int_{V}\left\{\int_{0}^{u_{\beta}} f_{\alpha} \mathrm{d} u_{\alpha}\right\} \mathrm{d} v=\int_{V} W \mathrm{~d} v \\
& \int_{\bar{S}}\left\{\int_{0}^{\bar{u}_{i}} F_{i} \mathrm{~d} u_{i}\right\} \mathrm{d} s+\int_{\bar{V}}\left\{\int_{0}^{\bar{u}_{j}} \bar{f}_{i} \mathrm{~d} u_{i}\right\} \mathrm{d} v=\int_{\bar{V}} \bar{W} \mathrm{~d} v
\end{aligned}
$$

In the above equations $W$ and $\bar{W}$ are the strain energy densities in configuration I and II, respectively, $u_{\beta}$ and $\bar{u}_{j}$ the displacements, $V$ and $\bar{V}$ the volumes of the bodies, and $S$ and $\bar{S}$ the surfaces. The outer shapes of I and II are, however, identical except for the difference in crack length, and since there is no loading on the crack faces we can set $S=\bar{S}$ in the surface integration. Since the crack is a slit with no thickness the two volumes are also identical, i.e. $\bar{V}=V$. Subtracting the second of Eqn. (12) from the first, now gives

$$
\begin{aligned}
& \int_{S}\left\{\int_{0}^{u_{\beta}} F_{\alpha} \mathrm{d} u_{\alpha}\right\} \mathrm{d} s-\int_{\bar{S}}\left\{\int_{0}^{\bar{u}_{j}} \bar{F}_{i} \mathrm{~d} u_{i}\right\} \mathrm{d} s+\int_{V}\left\{\int_{0}^{u_{\beta}} f_{\alpha} \mathrm{d} u_{\alpha}\right\} \mathrm{d} v-\int_{\bar{V}}\left\{\int_{0}^{\bar{u}_{j}} \bar{f}_{i} \mathrm{~d} u_{i}\right\} \mathrm{d} v \\
& \quad=\int_{V} W \mathrm{~d} v-\int_{\bar{V}} \bar{W} \mathrm{~d} v
\end{aligned}
$$


It now seems appropriate to assume that neither the surface tractions nor the body force on a material point change much from configuration I to configuration II, and the forces in configuration II will, therefore, be expressed as

$$
\begin{gathered}
\bar{F}_{i}\left(y_{j}\right)=\delta_{i \alpha}\left(F_{\alpha}\left(x_{\beta}\right)+\Delta F_{\alpha}\left(x_{\beta}\right)\right) \\
\bar{f}_{i}\left(y_{j}\right)=\delta_{i \alpha}\left(f_{\alpha}\left(x_{\beta}\right)+\Delta f_{\alpha}\left(x_{\beta}\right)\right)
\end{gathered}
$$

where $y_{j}=\delta_{j \beta} x_{\beta}$. Hence, both surface forces and body forces in configuration II are expressed in terms of the forces on the same material points in configuration I plus the increments $\Delta F_{\alpha}$ and $\Delta f_{\alpha}$. $S=\bar{S}$

If we look at the surface integral in (13), we get by help of (10) and (14) since

$$
\begin{aligned}
& \int_{S}\left\{\int_{0}^{u_{\beta}} F_{\alpha} \mathrm{d} u_{\alpha}\right\} \mathrm{d} s-\int_{\bar{S}}\left\{\int_{0}^{\bar{u}_{i}} \bar{f}_{i} \mathrm{~d} u_{i}\right\} \mathrm{d} v \\
& =\int_{S}\left\{\int_{0 .}^{u_{\beta}} F_{\alpha} \mathrm{d} u_{\alpha}-\int_{0}^{\delta_{j \beta}\left(u_{\beta}+\Delta u_{\beta}\right)} \delta_{i \alpha}\left(F_{\alpha}+\Delta F_{\alpha}\right) \mathrm{d} u_{i}\right\} \mathrm{d} s \\
& =-\int_{S}\left\{\int_{0}^{u_{\beta}} \Delta F_{\alpha} \mathrm{d} u_{\alpha}\right\} \mathrm{d} s-\int_{S} F_{\alpha} \Delta u_{\alpha} \mathrm{d} s
\end{aligned}
$$

In this expression for the difference in the work done by the surface tractions on the two configurations, the second term denotes the work done by the surface forces when the displacements are increased by the amount $\Delta u_{\alpha}$ and the first term denotes the energy released by the surface forces when going from configuration I to configuration II. A schematic representation of the two terms is given in Fig. 2. It should be noted that $\Delta u_{\alpha}$ in the surface integral is equal to the difference in physical displacement between corresponding points on the two surfaces. Since the crack is longer in configuration II than in configuration I, the surface forces are in general larger in configuration $I$ than in configuration II for equal displacements. $\Delta F_{\alpha}$ is, therefore, in general negative and the released energy positive. Hence, (15) states that the difference in the work done by the surface forces on the two configurations is

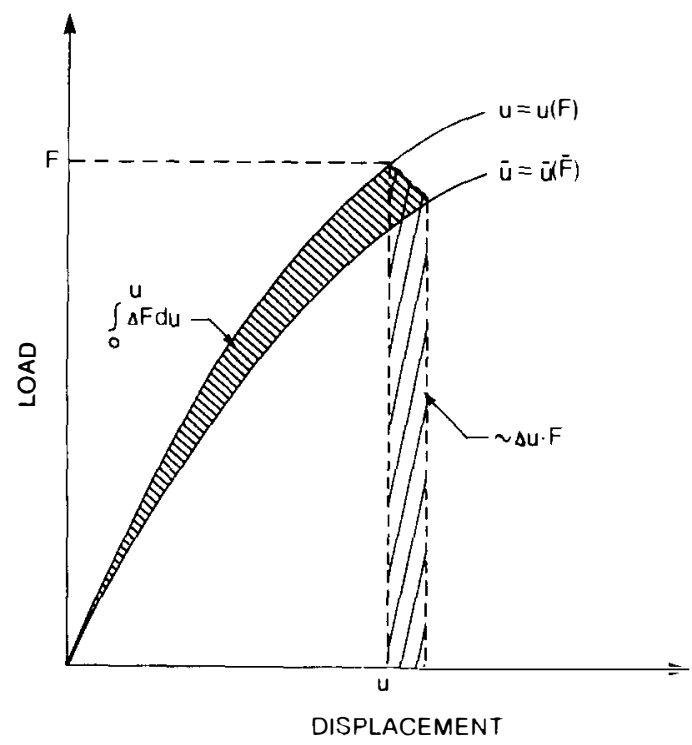

Figure 2. Schematic representation of work done by surface forces due to crack advance. 
equal to the released energy minus the work done in moving the surface forces the distance $\Delta u_{\alpha}$.

For the body force integrals in (13) we get similarly by help of (10) and (14)

$$
\begin{aligned}
& \int_{V}\left\{\int_{0}^{u_{\beta}} f_{\alpha} \mathrm{d} u_{\alpha}\right\} \mathrm{d} v-\int_{\bar{V}}^{\bar{u}_{j}}\left\{\bar{f}_{i} \mathrm{~d} u_{i}\right\} \mathrm{d} v \\
& =\int_{V}\left\{\int_{0}^{u_{\beta}} f_{\alpha} \mathrm{d} u_{\alpha}-\int_{0}^{\delta_{i \beta}\left(u_{\beta}+\Delta u p_{\beta}\right)} \delta_{i \alpha}\left(f_{\alpha}+\Delta f_{\alpha}\right) \mathrm{d} u_{i}\right\} \mathrm{d} v \\
& \quad \approx-\int_{V}\left\{\int_{0}^{u_{\beta}} \Delta f_{\alpha} \mathrm{d} u_{t x}\right\} \mathrm{d} v-\int_{V} f_{\alpha} \Delta u_{\alpha}^{m} \mathrm{~d} v
\end{aligned}
$$

The second term in (16) is now the work done by the body forces when displaced the distance $\Delta u_{\alpha}^{m}$ and the first term is the energy released by the body forces when going from configuration I to II. The physical displacement increment is given by (11) and is composed of the increments $\Delta u_{\alpha}$ and the term $-\left(\partial u_{\alpha} / \partial x_{\beta}\right) \Delta x_{\beta}$. Since $\partial u_{\alpha} / \partial x_{\beta}$ may be unbounded at the crack front, the last integral in (16) should be interpreted in the sense that a small region of radius $\rho$ around the crack front is excluded from the integration. The value of the integral is then taken as the limit value when $\rho \rightarrow 0$. The integral will have a finite value when the term $f_{\alpha}\left(\partial u_{\alpha} / \partial x_{\beta}\right) \rho^{2}$ approaches a finite value as $\rho \rightarrow 0$, hence the only requirement is that the value of $\partial u_{\alpha} / \partial x_{\beta}$ at most is of the order $1 / \rho^{2}$ for $\rho \rightarrow 0$.

The strains in configuration II at a point $\bar{y}_{k}$ are given by

$$
\overline{\boldsymbol{\epsilon}}_{i j}=\left.\frac{1}{2}\left(\frac{\partial \bar{u}_{i}}{\partial y_{j}}+\frac{\partial \bar{u}_{j}}{\partial y_{i}}\right)\right|_{\text {at } \bar{y}_{k}}
$$

By help of (7) and (6) we now get by neglecting all higher order terms

$$
\left.\frac{\partial \bar{u}_{i}}{\partial y_{j}}\right|_{\text {at } \bar{y}_{k}}=\left.\frac{\partial}{\partial x_{\beta}}\left\{\delta_{i \alpha}\left(u_{\alpha}+\Delta u_{\alpha}\right)\right\} \frac{\partial x_{\beta}}{\partial y_{j}} \approx \delta_{i \alpha} \delta_{j \beta}\left(\frac{\partial u_{\alpha}}{\partial x_{\beta}}+\frac{\partial \Delta u_{\alpha}}{\partial x_{\beta}}-\frac{\partial u_{\alpha}}{\partial x_{\delta}} \frac{\partial \Delta x_{\delta}}{\partial x_{\beta}}\right)\right|_{\text {at } x_{\gamma}}
$$

where $\bar{y}_{k}$ and $x_{y}$ are related through (3). The strains at $\bar{y}_{k}$ are then

$$
\left.\left.\bar{\epsilon}_{i j}\right|_{\text {at } \bar{y}_{k}} \approx \delta_{i \alpha} \delta_{j \beta}\left(\epsilon_{\alpha \beta}+\Delta \epsilon_{\alpha \beta}\right)\right|_{\text {at } x_{y}}
$$

where $\epsilon_{\alpha \beta}$ is the strain tensor at the point $x_{\gamma}$ in configuration I and

$$
\Delta \epsilon_{\alpha \beta}=\frac{1}{2}\left(\frac{\partial \Delta u_{\alpha}}{\partial x_{\beta}}+\frac{\partial \Delta u_{\beta}}{\partial x_{\alpha}}\right)-\frac{1}{2}\left(\frac{\partial u_{\alpha}}{\partial x_{\delta}} \frac{\partial \Delta x_{\delta}}{\partial x_{\beta}}+\frac{\partial u_{\beta}}{\partial x_{\delta}} \frac{\partial \Delta x_{\delta}}{\partial x_{\alpha}}\right)
$$

The shifters $\delta_{i \alpha}$ and $\delta_{j \beta}$ again only serve to shift the tensor quantities in the parentheses from the $x$-system to the $y$-system and do not change their magnitudes. Since the strain energy density is solely a function of the magnitude of the strain we must have that $W\left(\delta_{i \alpha} \delta_{i \beta} \epsilon_{\alpha \beta}\right) \equiv W\left(\epsilon_{\alpha \beta}\right)$ and the strain energy density in configuration II can be written as

$$
\left.\bar{W}\right|_{\text {at } \bar{y}_{k}}=\left.W\left(\epsilon_{\alpha \beta}\right)\right|_{\text {at } x_{\gamma}}+\frac{\partial W}{\partial \epsilon_{\alpha \beta}} \Delta \epsilon_{\alpha \beta}
$$

We will now restrict the material to be hyperelastic [7] and the stress-strain relationship can, therefore, be written

$$
\sigma_{\alpha \beta}=\frac{\partial W}{\partial \epsilon_{\alpha \beta}}
$$

Since the stress tensor is symmetric we obtain for the strain energy density in 
configuration II

$$
\left.\bar{W}\right|_{\text {at } \bar{y}_{k}}=\left.W\right|_{\text {at } x_{\gamma}}+\left.\sigma_{\alpha \beta}\left(\frac{\partial \Delta u_{\alpha}}{\partial x_{\beta}}-\frac{\partial u_{\alpha}}{\partial x_{\delta}} \frac{\partial \Delta x_{\delta}}{\partial x_{\beta}}\right)\right|_{\text {at } x_{\gamma}}
$$

With this expression for the strains in configuration II we can now evaluate the integrals of the strain energy densities on the right-hand side of (13). Since the strain energy density at $\bar{y}_{k}$ in configuration II is given as a function of the strain energy density at the corresponding location, $x_{\gamma}$, in configuration $\mathrm{I}$, it simplifies the integration if one takes advantage of the mapping in (3) and evaluates both integrals in the $x$-system. This will map the singularities of the two crack fronts into each other and facilitate taking the difference between the strain energies. We then obtain with (5) and (23) and by neglecting all higher order terms

$$
\begin{aligned}
\int_{V} W \mathrm{~d} v-\int_{\bar{V}} \bar{W} \mathrm{~d} v & =\int_{V}\{W-\bar{W} \operatorname{det}(J)\} \mathrm{d} v \\
& \approx \int_{V}\left\{\left(\sigma_{\alpha \beta} \frac{\partial u_{\alpha}}{\partial x_{\delta}}-W \delta_{\beta \delta}\right) \frac{\partial \Delta x_{\delta}}{\partial x_{\beta}}-\sigma_{\alpha \beta} \frac{\partial \Delta u_{\alpha}}{\partial x_{\beta}}\right\} \mathrm{d} v
\end{aligned}
$$

where $\delta_{\beta \delta}$ (both indices are Greek letters) denote Kronecker's delta. It should be noted that the body force integrals in (16) also could have been evaluated utilizing the mapping in (3). The results would have been the same, but the procedure would in that instance have led to a more cumbersome derivation.

Substituting Eqns. (15), (16), and (24) into (13) we finally obtain

$$
\begin{aligned}
G^{*}= & \int_{S} F_{\alpha} \Delta u_{\alpha} \mathrm{d} s+\int_{V} f_{\alpha} \Delta u_{\alpha}^{m} \mathrm{~d} v \\
& +\int_{V}\left\{\left(\sigma_{\alpha \beta} \frac{\partial u_{\alpha}}{\partial x_{\delta}}-W \delta_{\beta \delta}\right) \frac{\partial \Delta x_{\delta}}{\partial x_{\beta}}-\sigma_{\alpha \beta} \frac{\partial \Delta u_{\alpha}}{\partial x_{\beta}}\right\} \mathrm{d} v
\end{aligned}
$$

where the energy released between configuration I and II, $G^{*}$, is defined as

$$
G^{*}=-\int_{S}\left\{\int_{0}^{u_{\beta}} \Delta F_{\alpha} \mathrm{d} u_{\alpha}\right\} \mathrm{d} s-\int_{V}\left\{\int_{0}^{u_{\beta}} \Delta f_{\alpha} \mathrm{d} u_{\alpha}\right\} \mathrm{d} v
$$

The equilibrium equation for a small material volume, $\partial \sigma_{\alpha \beta} / \partial x_{\beta}+f_{\alpha}=0$, together with the divergence theorem gives for the last term in (25)

$$
\int_{V} \sigma_{\alpha \beta} \frac{\partial \Delta u_{\alpha}}{\partial x_{\beta}} \mathrm{d} v=\int_{S} \sigma_{\alpha \beta} n_{\beta} \Delta u_{\alpha} \mathrm{d} s+\int_{V} f_{\alpha} \Delta u_{\alpha} \mathrm{d} v
$$

where $n_{\beta}$ is the normal to the boundary. Again all the above integrals are evaluated by excluding a small region with radius $\rho$ around the crack front and letting $\rho \rightarrow 0$. The limiting values of the integral will exist is the stress and strain fieds are singular in such a way that $\sigma_{\alpha \beta} \rho$ and $\epsilon_{\alpha \beta} \rho$ remain bounded as $\rho \rightarrow 0$. Since $\sigma_{\alpha \beta} n_{\beta}$ is equal to the traction on the surface of the body we can finally by help of (27) and (11) write the expression for the released energy in the general 3-dimensional formulation

$$
G^{*}=\int_{V}\left\{\left(\sigma_{\alpha \beta} \frac{\partial u_{\alpha}}{\partial x_{\delta}}-W \delta_{\beta \delta}\right) \frac{\partial \Delta x_{\delta}}{\partial x_{\beta}}-f_{\alpha} \frac{\partial u_{\alpha}}{\partial x_{\beta}} \Delta x_{\beta}\right\} \mathrm{d} v
$$

Again it should be noted that this formulation is valid for a general anisotropic material and that no assumptions have been made about the direction of the crack propagation. It will, therefore, also be valid for shear lips and cracks growing off at an angle to the original crack plane. 


\section{Mappings and definition of the energy release rate}

Until now we have not been concerned with the form of the mapping, (3), which maps configuration I into configuration II. There are, of course, infinitely many mappings which will give the same crack extension $\Delta a$. Of specific interest for finite element application is, however, a scheme where $\Delta x_{\alpha}$ is constant over most of the body and only varies over a small region. This is the scheme that has been used by Hellen $[1,3]$ and Parks $[2,4,5]$ and it will briefly be described here. For simplicity we will only deal with a 2-D geometry, but the method is easily extended to 3-D configurations as well. Consider the crack geometry shown in Fig. 3a. To map this configuration into a configuration which has a crack length $a+\Delta a$, the body is divided into the three regions I, II, and III. In a finite element model region II would typically be one layer of elements. Let now $\Delta x_{\alpha} \equiv 0$ in region I, $\Delta x_{\alpha} \equiv \Delta a_{\alpha}$ in region III, and let $\Delta x_{\alpha}$ vary from 0 to $\Delta a_{\alpha}$ in region II. In the absence of body forces, the calculation of the released energy will only require the volume integration in (28) to be performed over one layer of elements (region II). As a special case region III can be shrunk into one point - the crack tip - with the resulting division shown in Fig. 3b. In this case the volume integration has to be performed over a small volume around the crack tip, only. In the presence of body forces the body force term in (28) had to be integrated both over region II and III for the mapping indicated in Fig. 3a, but only over the region II for the mapping shown in Fig. 3b.

In the general 3-dimensional case certain assumptions have to be made about the magnitude of $\Delta a$ along the crack front when calculating the released energy. In a test specimen a crack will grow all along the crack front and the energy release rate derived from the test will, therefore, represent some kind of average value. It seems appropriate to define the average value of the energy release rate by calculating the total energy released by a unit crack extension all along the crack front and divide it by the total length of the crack front. Referring to Fig. 4a the average energy release rate can then be defined as

$$
G_{\mathrm{av}}=\frac{G^{*}}{\Delta a \cdot l}=\frac{G^{*}}{A}
$$
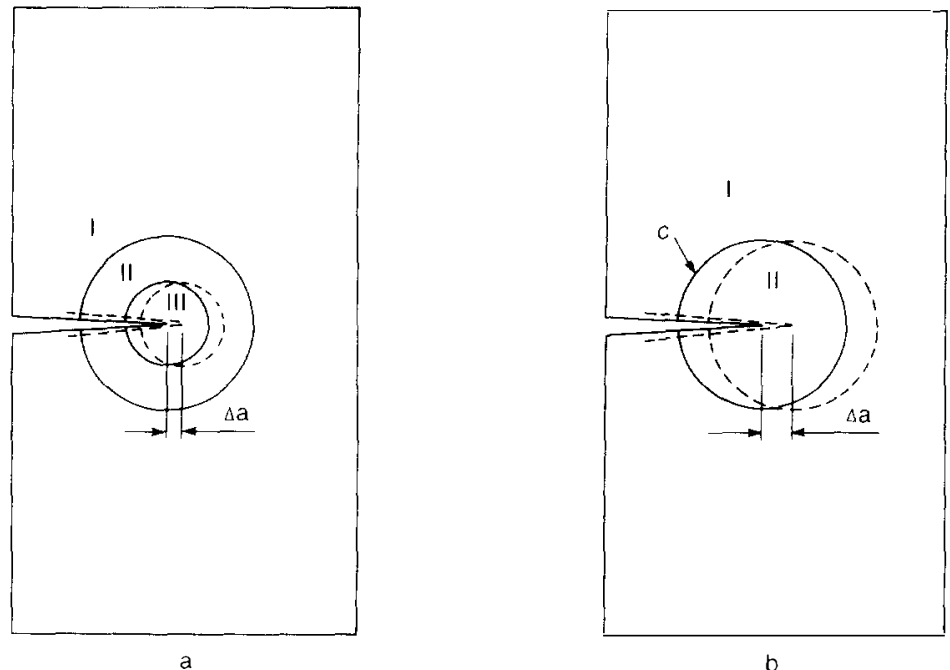

Figure 3. Typical modeling of crack advance. 

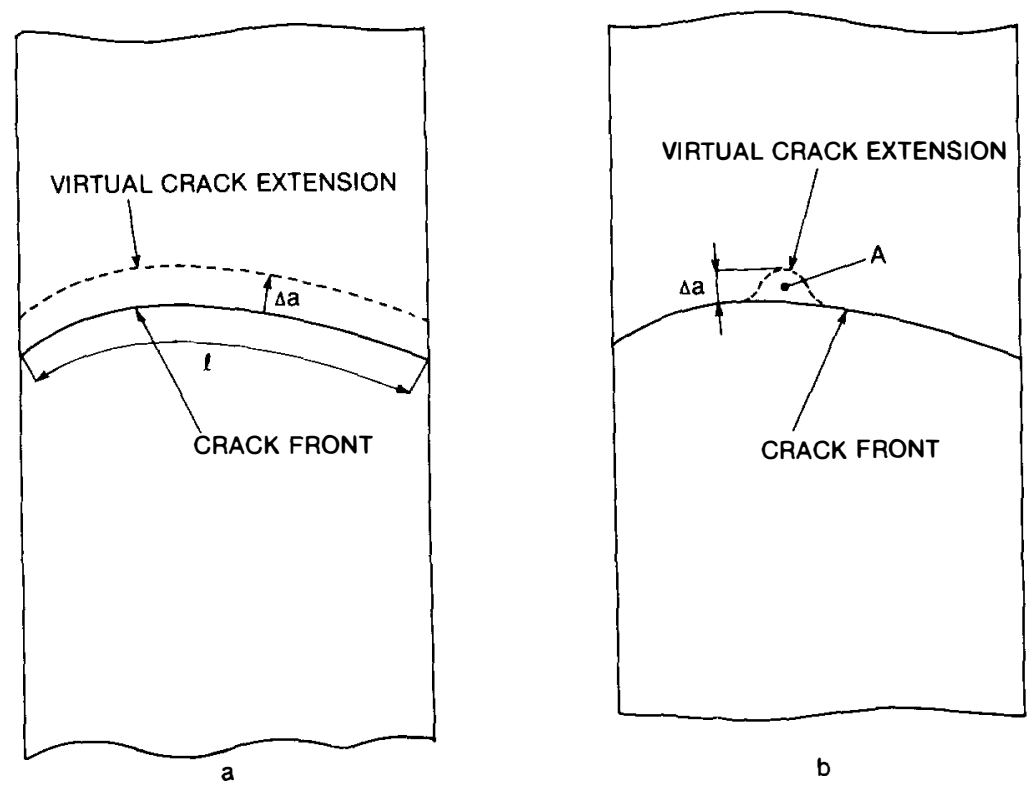

Figure 4. Virtual crack extensions for definition of average and local energy release rate.

where $G^{*}$ is the total energy released by the uniform crack extension $\Delta a$ and $A$ is the area covered by the virtual crack extension.

To define the local energy release rate, let us consider a crack extension which advances the crack locally at a given point on the crack front as sketched in Fig. 4b. If $A$ is the area of the virtual crack extension we will define the effective crack width as $b_{\text {eff }}=A / \Delta a$ and the local energy release rate can then be defined as

$$
G_{\text {local }}=\frac{G^{*}}{\Delta a \cdot b_{\text {eff }}}=\frac{G^{*}}{A}
$$

where $G^{*}$ is the total energy released by the localized crack extension.

In a typical finite element application the average energy release rate can then be found by advancing all node points on the crack front a distance $\Delta a$ and dividing the total released energy by the area of the virtual crack extension. By advancing one node at a time and calculating the area of the virtual crack extension from the finite element interpolation functions, the local energy release rate can be calculated according to (30).

\section{The energy release rate and the $J$-integral}

If we adopt for a planar crack in 3-D space the mapping indicated by Fig. $3 \mathrm{~b}$ and let $\Delta x \equiv \Delta a$ in region II, the crack will continue to propagate in the original crack plane. If we denote the volumes of the two regions by $V_{I}$ and $V_{I I}$ we get, since $\partial \Delta x / \partial x=0$ in $V_{\text {II }}$, that the released energy is

$$
G^{*}=\int_{V_{\mathrm{I}}}\left\{\left(\sigma_{\alpha \beta} \frac{\partial u_{\alpha}}{\partial x_{\delta}}-W \delta_{\beta \delta}\right) \frac{\partial \Delta x_{\delta}}{\partial x_{\beta}}-f_{\alpha} \frac{\partial u_{\alpha}}{\partial x_{\beta}} \Delta x_{\beta}\right\} \mathrm{d} v-\int_{V_{\mathrm{II}}} f_{\alpha} \frac{\partial u_{\alpha}}{\partial x_{\beta}} \Delta x_{\beta} \mathrm{d} v
$$


Using the divergence theorem (31) can also be written

$$
\begin{aligned}
G^{*}= & \int_{S_{1}} \eta_{\beta}\left(\sigma_{\alpha \beta} \frac{\partial u_{\alpha}}{\partial x_{\beta}}-W \delta_{\beta \delta}\right) \Delta x_{\delta} \mathrm{d} s \\
& -\int_{V_{1}}\left\{\frac{\partial \sigma_{\alpha \beta}}{\partial x_{\beta}} \frac{\partial u_{\alpha}}{\partial x_{\delta}}+\sigma_{\alpha \beta} \frac{\partial^{2} u_{\alpha}}{\partial x_{\delta} \partial x_{\beta}}-\frac{\partial W}{\partial x_{\beta}}+f_{\alpha} \frac{\partial u_{\alpha}}{\partial x_{\delta}}\right\} \Delta x_{\delta} \mathrm{d} v-\int_{V_{11}} f_{\alpha} \frac{\partial u_{\alpha}}{\partial x_{\beta}} \Delta x_{\beta} \mathrm{d} v
\end{aligned}
$$

where $S_{1}$ is the surface of region I including the surface separating region I and II. In this expression the volume integral over region $I$ is, however, identical to zero because of the equilibrium equation, $\left(\partial \sigma_{\alpha \beta} / \partial x_{\beta}\right)+f_{\alpha}=0$, together with (22). Since $n_{\alpha}$ is the normal to the surface and $\Delta x_{\alpha}$, except on the boundary to region II, necessarily must be parallel to the surface it is seen that the last term, $W n_{\beta} \Delta x_{\beta}$, in the surface integral is zero everywhere on the outer boundary. Now let the surface traction be defined by $t_{\alpha}=\sigma_{\alpha \beta} n_{\beta}$ and let us assume that on the surface of region I, except on the boundary to region II, either $t_{\alpha}=0$, i.e., there is no loading on this part of the surface, or that $\Delta x_{\delta}=0$. This last condition is always fulfilled except where a crack intersects a free surface in which case the mapping schematically shown in Fig. $3 \mathrm{~b}$ also will affect the outer surface. If one of these two conditions hold on the outer surface of region I the released energy can be written as

$$
G^{*}=\int_{C}\left(t_{\alpha} \frac{\partial u_{\alpha}}{\partial x_{\delta}} \Delta x_{\delta}-W \eta_{\delta} \Delta x_{\delta}\right) \mathrm{d} s-\int_{V_{11}} f_{\alpha} \frac{\partial u_{\alpha}}{\partial x_{\beta}} \Delta x_{\beta} \mathrm{d} v
$$

where $C$ is the surface separating region I and II. It should, however, be noted that (33) also is valid if certain symmetry conditions are fulfilled as, for example, under plane strain conditions. If the geometry of a plane strain problem is defined in the $x_{1}-x_{2}$ plane we will have $\Delta x_{3}=t_{1}=t_{2}=0$ on symmetry plane, but since $\partial u_{3} / \partial x_{1}=$ $\partial u_{3} / \partial x_{2}=0$ under plane strain conditions the outer surface integral will also vanish when applied to a slice of a body.

If $\Delta a_{\delta}$ represents a unit crack extension and $G^{*}$ is evaluated per unit length of the crack front, (33) can be considered the general 3-dimensional form of the $J$-integral. Since $\Delta x_{\delta}=\Delta a_{\delta}$ is constant in region II and, therefore, also on its boundary, the quantity can also be written as

$$
G^{*}=J_{\delta} \Delta a_{\delta}
$$

where

$$
J_{\delta}=\int_{C}\left(t_{\alpha} \frac{\partial u_{\alpha}}{\partial x_{\delta}}-W n_{\delta}\right) \mathrm{d} s-\int_{V_{11}} f_{\alpha} \frac{\partial u_{\alpha}}{\partial x_{\delta}} \mathrm{d} v
$$

In the absence of body forces the expression in (35) was derived for 2-D applications by Eshelby [8, 12] and Rice [9] and for 3-D applications by Knowles and Sternberg [10]. The body force term of (35) was included by Sakata et al. [11] for the 2-D formulation. It is, however, seen that (35) by itself is only valid for the specialized case where the crack is lined up with a coordinate axis and if the crack continues to extend in the crack plane.

If a numerical analysis is carried out by means of the finite element method, the volume integral formulation for calculating the released energy, (28), is much preferred over the surface integral formulation Eqns. (34) and (35). The former is a natural extension of the volume integrals already carried out in a finite element analysis while the latter method gives rise to additional difficulties in defining the surface over which to integrate and in performing the actual surface integration. This is especially true when higher order finite elements are used. 


\section{Summary}

An analytical expression for the energy released by an arbitrary crack propagation in a general 3-D crack configuration has been derived from a continuum mechanics viewpoint. Previous expressions for the energy release rate by the virtual crack extension method were based on numerical considerations [1-5] and were, therefore, only valid for that specific numerical method. The expression presented in this paper has, however, general validity. When working in 3-D fracture mechanics it seems appropriate to give both an average and local definition of the energy release rate. These definitions are suggested in the paper. It is also shown that the general 3-D expression for the energy release rate under certain simplifying assumptions of the fracture behavior reduces to the 3-D form of the $J$-integral.

The present expression for the energy release rate can be used effectively in finite element applications since it can take full advantage of the numerical integration schemes already available in a finite element program. An application of the method is given in [6] which describes elastic-plastic analyses of a standard and a side-grooved compact specimen.

\section{REFERENCES}

[1] T.K. Hellen, "The Finite Element Calculations of Stress Intensity Factors Using Energy Techniques," 2nd International Conference on Structural Mechanics in Reactor Technology (SMiRT), Paper G5/3, Berlin (1973).

[2] D.M. Parks, International Journal of Fracture 10, No. 4 (1974) 487-502.

[3] T.K. Hellen, Intemational Joumal for Numerical Methods in Engineering 9 (1975) 187.

[4] D.M. Parks, Computer Methods in Applied Mechanics and Engineering 12 (1977) 353.

[5] D.M. Parks, in Proceedings of the First International Conference on Numerical Methods in Fracture Mechanics, Swansea (1978) 464.

[6] H.G. deLorenzi and C.F. Shih, "3-D Elastic-Plastic Investigation of Fracture Parameters in Side Grooved Compact Specimen," accepted for publication by International Joumal of Fracture.

[7] C. Truesdell, in Encyclopedia of Physics, ed. by S. Flügge, Springer-Verlag, Berlin (1960) sec. 303.

[8] J.D. Eshelby, Solid State Physics, Vol. 3, Academic Press, New York (1956).

[9] J.R. Rice, Joumal of Applied Mechanics (June 1968) 379.

[10] J.K. Knowles and E. Sternberg, Archive for Rational Mechanics and Analysis 44, No. 3 (1972) 187.

[11] M. Sakata, S. Aoki and K. Ishii, in Proceedings of International Conference on Fracture Mechanics and Technology, ed. G.C. Sih and C.L. Chow, Vol. 1, Sijthoff and Noordhoff Publishers (1977) 515.

[12] J.D. Eshelby, in Inelastic Behavior of Solids, edited by M.F. Kanninen et al., McGraw-Hill, New York (1970). 